\title{
Optical sectioning with a low-coherence phase-shifting digital holographic microscope
}

\author{
Yu-Chih Lin, ${ }^{1}$ Chau-Jern Cheng, ${ }^{1, *}$ and Ting-Chung Poon ${ }^{2}$ \\ ${ }^{1}$ Institute of Electro-Optical Science and Technology, National Taiwan Normal University, Taipei 11677, Taiwan \\ ${ }^{2}$ Bradley Department of Electrical and Computer Engineering, Virginia Tech, Blacksburg, Virginia 24060, USA \\ *Corresponding author: cjcheng@ntnu.edu.tw
}

Received 28 July 2010; revised 28 October 2010; accepted 31 October 2010; posted 2 November 2010 (Doc. ID 132453); published 6 December 2010

\begin{abstract}
The properties of a low-coherence phase-shifting digital holographic microscope are first studied and analyzed. We then demonstrate en face imaging with transverse resolution of $3 \mu \mathrm{m}$ and axial resolution of $10 \mu \mathrm{m}$ through a thickness of $300 \mu \mathrm{m}$ onion membrane. In addition, with the above said resolutions, optical sectioning of the eye and spine of a live zebra fish has been demonstrated. To the best of our knowledge, this is the first time that a short coherence phase-shifting holographic microscope has been applied to the internal structure visualization of a biological specimen under an in vivo environment. (C) 2010 Optical Society of America
\end{abstract}

OCIS codes: $\quad 090.1995,110.4980$.

\section{Introduction}

One of the challenges in three-dimensional (3D) microscopy has been to overcome an out-of-focus haze. Hence, optical sectioning has been an important topic in biomedical applications. Optical coherence tomography (OCT) [1] and confocal microscopy [2] have been the main-stream techniques routinely used for sectioning. In OCT, low-coherence light is used to obtain the interference between a reference wave and the light scattered by a transverse section of the specimen with matching optical path length within the coherence length of the light source. In contrast, in confocal microscopy, a single point of the specimen is imaged to a pinhole placed in front of a photodetector and, thereby, the out-of-focus energy is rejected from other transverse sections. However, both techniques have a common drawback in that the data are collected one point at a time and each transverse section requires a precise twodimensional scan. An alternative technique for 3D imaging is holography [3]. Holography is capable of capturing a volume of specimen with high resolu-

0003-6935/11/070B25-06\$15.00/0

(C) 2011 Optical Society of America tion. Because of the advent of high-resolution CCDs and powerful digital processing, digital holography has become one of the dominant techniques for biomedical applications, as well as for industrial inspection applications [4]. The use of digital holography and postprocessing to achieve sectioning has been investigated rigorously in recent years [ $\underline{5}-11]$, mainly within the context of optical scanning holography [12]. Recently, digital holography with a low-coherence source has been investigated in microscopy, and it shows the ability to perform optical sectioning [13-16]. The use of digital holography for optical sectioning has also been demonstrated by a so-called wavelength-scanning digital interference holography method [17-19]. In another application of a lowcoherence holographic system, a phase target embedded in a light-scattering medium for the investigation of data protection techniques has been studied [20]. Some recent investigations of low-coherence holography include fingerprint biometry applications [21] and optical sectioning using a femtosecond-laser source [22].

In this paper, we first investigate the properties of a low-coherence phase-shifting holography (PSH). Reconstruction errors in amplitude and phase resulting from a low-coherent source against a coherent 
source are investigated within the context of phaseshifting digital holography [23]. We then demonstrate a low-coherence phase-shifting holographic system using a ytterbium-doped fiber amplifier (YDFA) as the light source. Optical sectioning through a union membrane is first demonstrated. We then show optical sectioning of the eye and spine of a live zebra fish as examples to demonstrate the usefulness of the technique in 3D microscopy.

\section{Principle and Properties of a Low-Coherence PSH}

We first discuss the working principle of a phaseshifting digital holographic microscope (DHM) equipped with a low-coherence light source. The object is illuminated by a low-coherence source. The reflected beam $\left(I_{O}\right)$ from the object and a reference beam $\left(I_{R}\right)$ are interfered and collected by a CCD, as shown in Fig. 1.

The intensity of the four phase-shifted interference fringes presented as digital holograms can thus be written as

$$
\begin{aligned}
I_{D n} \propto & I_{O}^{2}+I_{R}^{2} \\
& +2\left|E_{O} \| E_{R}\right|\left|\operatorname{sinc}\left[(n-1) \frac{\Delta \lambda}{4 \lambda}\right]\right| \cos [\phi \\
& \left.+(n-1) \frac{\pi}{2}\right], \\
n & =1,2,3,4,
\end{aligned}
$$

where the symbols $E_{O}$ and $E_{R}$ are the complex amplitude of the reflected and reference waves on the CCD plane, respectively. The function $\operatorname{sinc}(\cdot)=$ $\sin (\cdot) /(\cdot)$ in Eq. (1) is a normalized mutual coherence function as we have considered the spectral shape of the low-coherence light source as a rectangular shape [24]. The normalized mutual coherence function is a function of the index $n$, which indicates that we change the position of the piezoelectric transducer (PZT)-driven mirror to shift several steps of the phase of the reference beam to perform the phaseshifting process. This movement of the PZT-driven mirror will cause the optical path difference between the object and the reference arms so that the fringe contrast of the hologram will be influenced. The symbols $\lambda$ and $\Delta \lambda$ are the center wavelength and the spectral bandwidth of the light source, respectively. The symbol $\phi=\frac{\pi}{\lambda}\left(l_{s}-l_{r}\right)$ represents the interference phase information between the object and the reference waves. $l_{s}$ denotes the distance between the object and the center of the beam splitter (BS). $l_{r}$ is the distance between the reference mirror and the center of the beam splitter and, by changing its distance, we can realize the different phase shifts between the object wave and the reference wave. By using the phase-shifting procedure and Eq. (1), we can derive the complex field $E_{0}=\left|E_{0}\right| e^{j \phi}$ on the CCD plane, where the phase of the complex field is written as

$$
\phi=\tan ^{-1}\left\{\frac{I_{D 4}-I_{D 2}}{I_{D 1}-I_{D 3}} \frac{\left[\operatorname{sinc}\left(\frac{\Delta \lambda}{2 \lambda}\right)+1\right]}{\left[\operatorname{sinc}\left(\frac{3 \Delta \lambda}{4 \lambda}\right)+\sin c\left(\frac{\Delta \lambda}{4 \lambda}\right)\right]}\right\},
$$

and the amplitude of the complex field is given by

$$
\left|E_{0}\right|=\frac{\sqrt{\left\{\left(I_{D 4}-I_{D 2}\right)\left[\operatorname{sinc}\left(\frac{\Delta \lambda}{2 \lambda}\right)+1\right]\right\}^{2}+\left\{\left(I_{D 1}-I_{D 3}\right)\left[\operatorname{sinc}\left(\frac{3 \Delta \lambda}{4 \lambda}\right)+\operatorname{sinc}\left(\frac{\Delta \lambda}{4 \lambda}\right)\right]\right\}^{2}}}{2\left[\operatorname{sinc}\left(\frac{\Delta \lambda}{2 \lambda}\right)+1\right]\left[\operatorname{sinc}\left(\frac{3 \Delta \lambda}{4 \lambda}\right)+\operatorname{sinc}\left(\frac{\Delta \lambda}{4 \lambda}\right)\right]}
$$

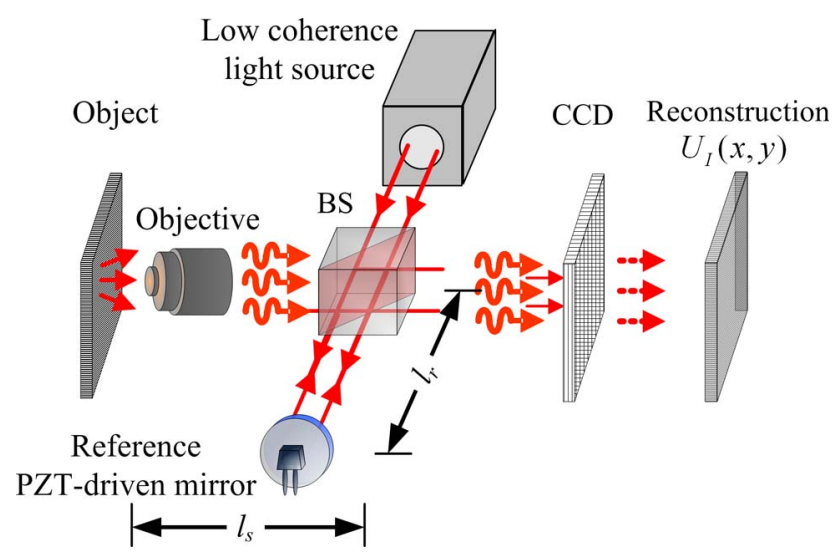

Fig. 1. (Color online) Setup of a low-coherence phase-shifting digital holographic microscope. assuming $\left|E_{r}\right|=1$. Note that, in the above expressions, we have included the normalized coherence function. Holographic reconstruction can now be done by performing Fresnel diffraction of $E_{0}=$ $\left|E_{0}\right| e^{j \phi}$ through a reconstruction distance $d_{O}[25]$ as

$$
U_{I}(x, y)=e^{j \frac{k}{2 d_{o}}\left(x^{2}+y^{2}\right)} \iint\left\{E_{0} e^{j_{2 d_{o}}\left(\xi^{2}+\eta^{2}\right)} e^{j \frac{2 \pi}{\lambda d_{0}}(x \xi+y \eta)}\right\} \mathrm{d} \xi \mathrm{d} \eta,
$$

where $(x, y)$ and $(\xi, \eta)$ are the coordinates of the reconstruction plane and the hologram plane, respectively, and the integration is over the area of the CCD. As indicated in Eq. (4), by invoking the linearity of the wave-propagation phenomenon, we can 
approximate the Fresnel diffraction as indicated in Eq. (4) for nonmonochromatic reconstruction when a sufficiently narrow bandwidth source is utilized $(\Delta \lambda / \lambda \ll 1)[26]$. To investigate the effect of low coherence on reconstruction, we consider $E_{0}=\left|E_{0}\right| e^{j \phi}=(A+\Delta A) e^{j(\phi+\Delta \phi)}$, where the terms $\Delta A$ and $\Delta \phi$ are the errors due to the effect of low coherence. When the errors go to zero, we have $A e^{j \phi}$ due solely to a complete coherence case, i.e., when the illumination of the object is purely coherent. Hence, the reconstruction due to low coherence can be written as

$$
\begin{aligned}
U_{I}(x, y)= & e^{j \frac{k}{2 d_{o}}\left(x^{2}+y^{2}\right)} \iint\{(A \\
& \left.+\Delta A) e^{j(\phi+\Delta \phi)} e^{j \frac{k}{2 d_{o}}\left(\xi^{2}+\eta^{2}\right)} e^{j \frac{2 \pi}{\lambda d_{o}}(x \xi+y \eta)}\right\} \mathrm{d} \xi \mathrm{d} \eta,
\end{aligned}
$$

and $U_{I}^{C}(x, y)$ is the reconstruction image in the case of a coherent light source, given by

$$
U_{I}^{C}(x, y)=e^{j \frac{k}{2 d_{o}}\left(x^{2}+y^{2}\right)} \iint\left\{A e^{j \phi} e^{j \frac{k}{2 d_{O}}\left(\xi^{2}+\eta^{2}\right)} e^{j \frac{2 \pi}{\lambda d_{O}}(x \xi+y \eta)}\right\} \mathrm{d} \xi \mathrm{d} \eta .
$$

The phase and amplitude error terms are given by

$$
\begin{aligned}
\Delta \phi= & \tan ^{-1}\left\{\frac{I_{D 4}-I_{D 2}}{I_{D 1}-I_{D 3}} \frac{\left[\operatorname{sinc}\left(\frac{\Delta \lambda}{2 \lambda}\right)+1\right]}{\left.\operatorname{sinc}\left(\frac{3 \Delta \lambda}{4 \lambda}\right)+\operatorname{sinc}\left(\frac{\Delta \lambda}{4 \lambda}\right)\right]}\right\} \\
& -\tan ^{-1}\left\{\frac{I_{D 4}-I_{D 2}}{I_{D 1}-I_{D 3}}\right\},
\end{aligned}
$$

$100 \mathrm{~nm}$. The value of $\Delta \lambda / \lambda \leq 0.094$ is much less than 1 so that the Fresnel diffraction of Eqs. (5)-(8) are still valid and can be directly applied to nonmonochromatic reconstruction. The size of the input image and the hologram are set to $200 \times 200$ pixels. For simplicity, the phase information of the input image is assumed to be a constant as a reference level. The reconstruction $U_{I}^{C}(x, y)$ with a coherent light source is shown in Fig. 2(a), and the reconstruction under the low-coherence light source is shown in Fig. 2(b). The reconstruction errors, which are derived from the difference between Eqs. (5) and (6), as a function of the spectral bandwidth of the light source, is also plotted in Figs. 2(c) and 2(d). One can see that the reconstruction errors are still minor as the spectral bandwidth increases up to $100 \mathrm{~nm}$ in phase-shifting DHM. We have also investigated the use of Gaussian and Lorentzian distribution shapes. As it turns out, the amplitude and phase errors from the rectangular shape of the spectral distribution are lower than those from the Gaussian and Lorentzian distribution shapes.

\section{Experiments and Results}

The experimental setup of a low-coherence phaseshifting digital holographic microscope for measuring sectional images is shown in Fig. 3(a). It mainly consists of a modified Michelson interferometer with a PZT-driven mirror in the reference arm and a high resolution CCD sensor (pixel number, $1280 \times 1024$; pixel size, $5.2 \mu \mathrm{m} \times 5.2 \mu \mathrm{m}$ ). The motorized linear stage was moved longitudinally step by step to different depths of the sample to capture holograms at different layers. Then, the PZT-driven mirror was used to achieve the phase-shifting algorithm for hologram recording with four-step phase intervals of $0^{\circ}, 90^{\circ}$, $180^{\circ}$, and $270^{\circ}$ for each layer. The objective is $10 \times$

$$
\begin{aligned}
\Delta A= & \frac{\sqrt{\left\{\left(I_{D 4}-I_{D 2}\right)\left[\operatorname{sinc}\left(\frac{\Delta \lambda}{2 \lambda}\right)+1\right]\right\}^{2}+\left\{\left(I_{D 1}-I_{D 3}\right)\left[\operatorname{sinc}\left(\frac{3 \Delta \lambda}{4 \lambda}\right)+\operatorname{sinc}\left(\frac{\Delta \lambda}{4 \lambda}\right)\right]\right\}^{2}}}{2\left[\operatorname{sinc}\left(\frac{\Delta \lambda}{2 \lambda}\right)+1\right]\left[\operatorname{sinc}\left(\frac{3 \Delta \lambda}{4 \lambda}\right)+\operatorname{sinc}\left(\frac{\Delta \lambda}{4 \lambda}\right)\right]} \\
& -\frac{\sqrt{\left(I_{D 4}-I_{D 2}\right)^{2}+\left(I_{D 1}-I_{D 3}\right)^{2}}}{4},
\end{aligned}
$$

respectively. Note that the image difference between the reconstructions $U_{I}^{C}(x, y)$ and $U_{I}(x, y)$ can thus be deduced theoretically from Eqs. (5)-(8). According to Eqs. (5) and (6), we simulate the effects on the reconstruction image under a low-coherence light source and complete coherence, respectively, and the simulation results are shown in Fig. 2. Assume that the center wavelength of the low-coherence light source is $1060 \mathrm{~nm}$ and the maximum spectral bandwidth is with a NA of 0.25 , corresponding to transverse resolution of about $3 \mu \mathrm{m}$. The low-coherence light source is a YDFA, whose spectral profile is shown in Fig. 3(b). The center wavelength is about $1060 \mathrm{~nm}$, and the spectral bandwidth is $50 \mathrm{~nm}$ at full width at half-maximum. The coherence gating length, $\lambda^{2} / \Delta \lambda$, determines the thickness of the sectional layer, which was estimated to be about $15 \mu \mathrm{m}$. In the experiment, we embedded a USAF 1951 positive 


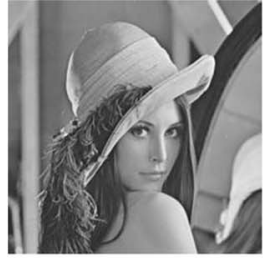

(a)

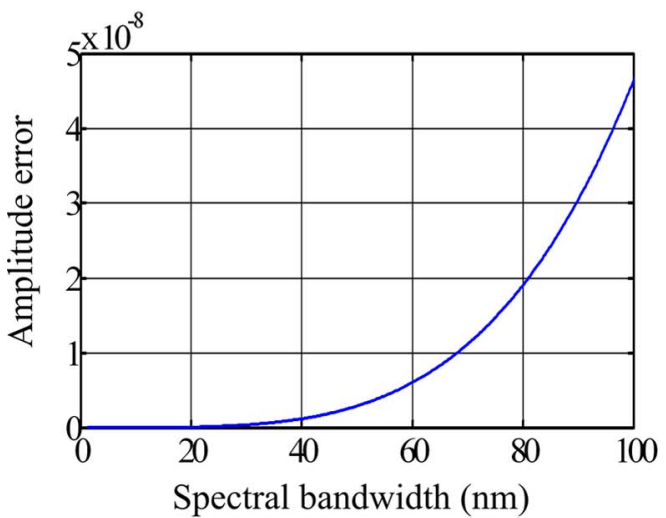

(c)

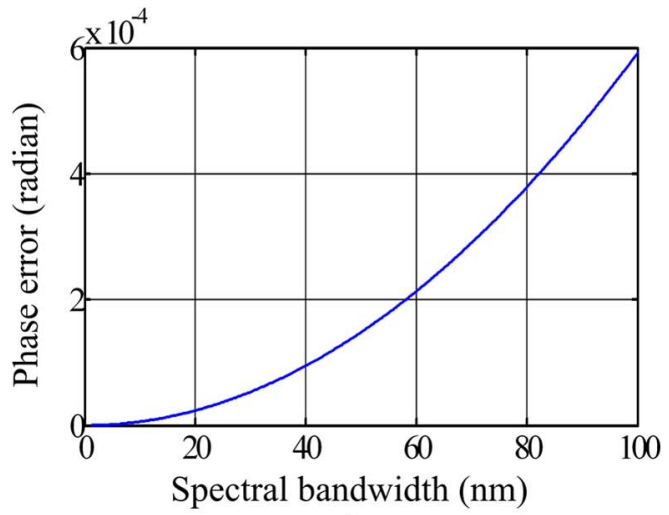

(d)

Fig. 2. (Color online) Influences of phase-shifting and low coherence on reconstruction (a) under a coherent light source and (b) with a $100 \mathrm{~nm}$ spectral bandwidth light source. (c) Errors in the reconstructed amplitude map under different spectral bandwidths and (d) errors in the reconstructed phase map under different spectral bandwidths.

resolution target into an onion membrane, as shown in Fig. 4(a). By coherent gating, only the particular optical path that fits the interference condition can generate the hologram fringes in the CCD camera; an en face sectional image can thus be reconstructed through numerical calculations at different depths of the sample. Reconstructions at different depths of the onion membrane are shown in Figs. 4(b)-4(d). The result of Fig. 4(b) is the case of a coherent light source; the resolution target cannot be obtained through the onion membrane and the reconstructed en face image of the onion is blurred due to the speckle noise from the highly scattering medium. Using the low-coherence light source, one can obtain a clearer onion surface image, as shown in Fig. 4(c), and the resolution target information can be

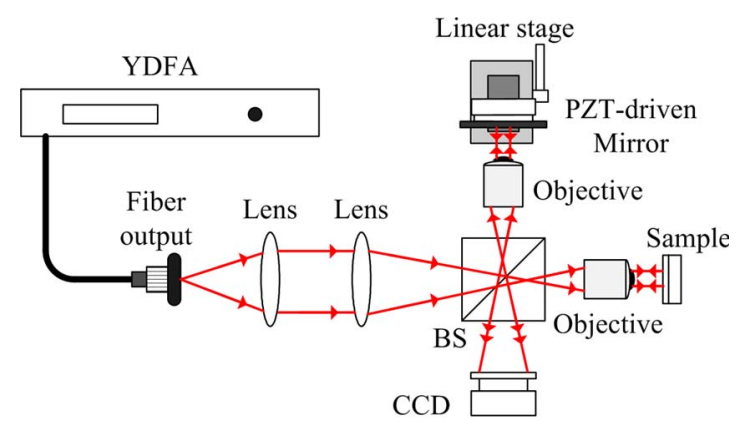

(a)

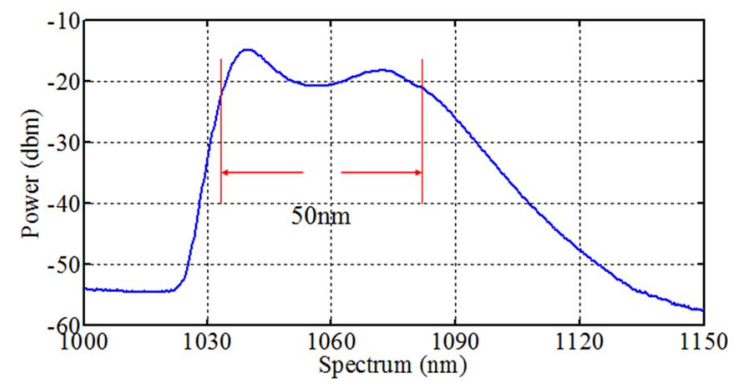

(b)

Fig. 3. (Color online) (a) Experimental setup of a low-coherence phase-shifting DHM and (b) spectral profile of the YDFA.

reconstructed under the onion surface, as shown in Fig. 4(d). We can recognize group 7 elements 4 on the target. These results can achieve a field of view of about $300 \mu \mathrm{m} \times 250 \mu \mathrm{m}$ with lateral resolution of about $3 \mu \mathrm{m}$. This means that one can rapidly measure an en face sectional image with micrometer

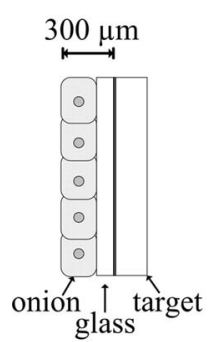

(a)

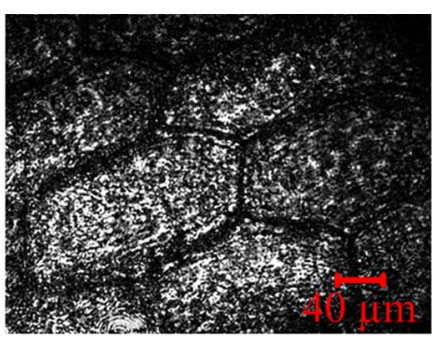

(b)

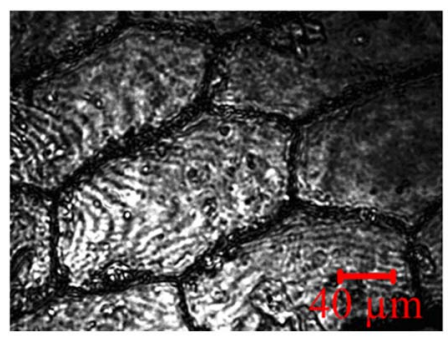

(c)

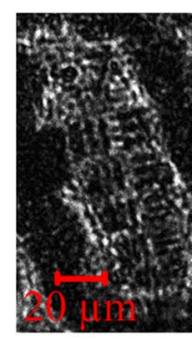

(d)
Fig. 4. (Color online) En face images of the onion membrane and USAF 1951 resolution target: (a) onion membrane and target configuration, (b) reconstructed surface image of the onion membrane by a coherent light source, (c) reconstructed surface image of the onion membrane by a low-coherence light source, and (d) sectional image of USAF 1951 resolution target under the onion surface. 
spatial resolution with the low-coherence digital holographic microscope.

In the next sets of experiments, we use a live zebra fish as a specimen. Zebra fish have become attractive vertebrate models in the study of vertebrate development due partly to the transparency of their embryos for optical inspection [27]. In our preliminary experiments with zebra fish, we attempt to image its eye and spine to demonstrate the effectiveness of our constructed low-coherence phase-shifting holographic microscope. In particular, we intend to demonstrate optical sectioning of the constructed microscope. Figure 5 shows the image of a zebra fish. The zebra fish has been tranquilized under anes-

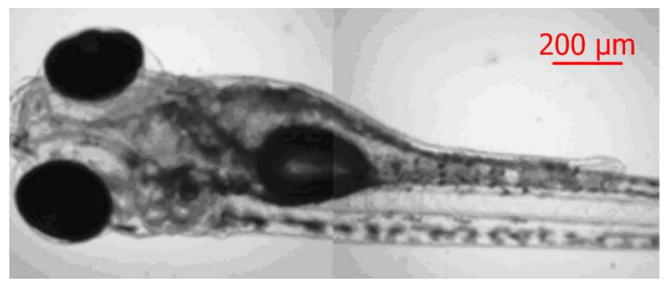

Fig. 5. (Color online) Image of a zebra fish.

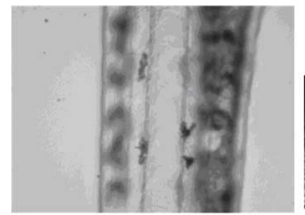

(a)

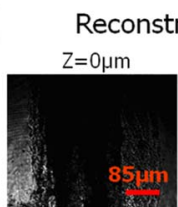

(b)

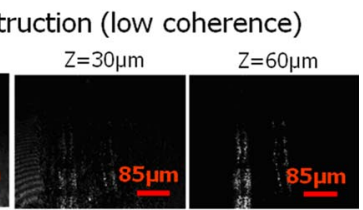

(c) (d)
Fig. 6. (Color online) (a) Portion of the tail to be holographically recorded. (b)-(d) Optical sections at three different sections.

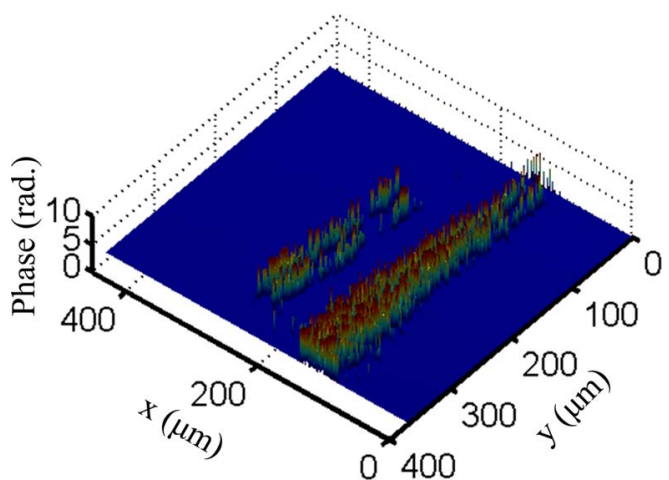

Fig. 7. (Color online) Phase plot at the section $z=60 \mu \mathrm{m}$ shown in Fig. $\underline{6(\mathrm{~d})}$.

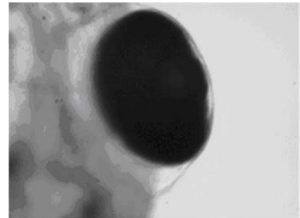

(a)

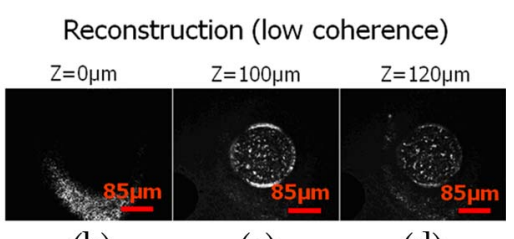

(b) (c) (d)
Fig. 8. (Color online) (a) Eye of the zebra fish. (b)-(d) Optical sections through a depth of $120 \mu \mathrm{m}$.

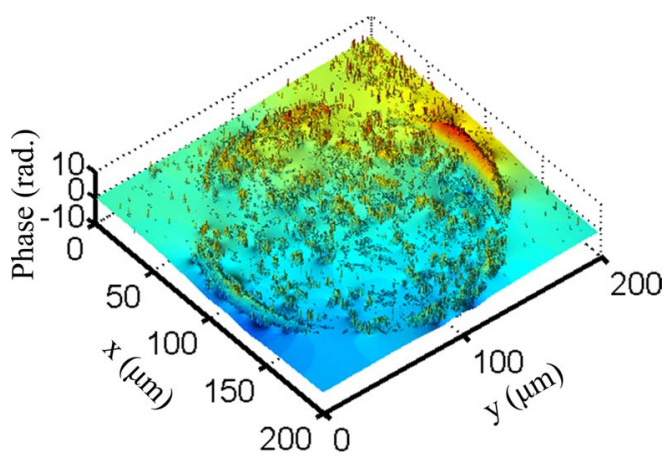

Fig. 9. (Color online) Phase plot of the section shown in Fig. 8(c).

thetic, and we used 2.5 frames/s to capture the holograms. Figure 6(a) shows a portion of the tail of the zebra fish where we capture the holograms, and Figs. 6(b)-6(d) show three optical sections. Clearly, in Fig. 6(d), we see the outline of the spine at that section. Figure 7 shows the phase plot at the section $z=60 \mu \mathrm{m}$, where the spine is clearly outlined. Next, in Fig. 8(a), we show the eye of the zebra fish. Figures $\overline{8(\mathrm{~b})}-8(\mathrm{~d})$ show optical sectioning to a depth of $120 \mu \mathrm{m}$. In Fig. 8(c), we clearly show the shape of the eyeball, and Fig. 9 shows the phase plot of Fig. 8(c). Because of the limited dynamic range of the $\overline{\mathrm{CCD}}$ camera (8 bits; gray levels, 0-255), we can only detect the signals above gray level 5. A little amplitude threshold at about gray level 5 was applied to reject the zeros of the pixel in the digital hologram for the phase-shifting process and hologram reconstruction. This threshold effect leads to a sharp phase map, as shown in the tail and the eye phase images in Figs. $\underline{7}$ and $\underline{9}$, respectively.

\section{Conclusions}

We have proposed and demonstrated a noncontact measurement technique for full field sectional en face imaging through a light-scattering medium by use of a low-coherence phase-shifting digital holographic microscope. In addition, optical sectioning experiments on a live zebra fish have been successfully demonstrated. The effects of using low coherence on reconstruction are also investigated. We found that reconstruction errors when using the spectral bandwidth of a low-coherent light up to $100 \mathrm{~nm}$ are negligible as compared to the case of using a coherent source for phase-shifting operations. We have demonstrated that low-coherence phase-shifting DHM offers the possibility of high-resolution 3D sectional imaging of a specimen by coherence gating and the technique can relax the restriction of the numerical aperture and the focusing mechanism of an objective. In the case of an ultra-wideband source, such as a supercontinuum of spectral bandwidth of about $500 \mathrm{~nm}$, very short coherence gating length of the order of $1 \mu \mathrm{m}$ is possible. We plan to look into such implementation for biomedical applications.

This work is financially supported by the National Science Council, Taiwan (NSCT), under 
contracts NSC-98-2221-E-003-006 and NSC-992221-E-003-010-MY3.

\section{References}

1. D. Huang, E. A. Swanson, C. P. Lin, J. S. Schuman, W. G. Stinson, W. Chang, M. R. Hee, T. Flotte, K. Gregory, C. A. Puliafito, and J. G. Fujimoto, "Optical coherence tomography," Science 254, 1178-1181 (1991).

2. T. Wilson and C. Sheppard, Theory and Practices of Scanning Optical Microscopy (Academic, 1984).

3. D. Gabor, "Holographic model of temporal recall," Nature 217, 584 (1968).

4. T.-C. Poon, ed., Digital Holography and Three-Dimensional Display: Principles and Applications (Springer, 2006).

5. T. Kim, "Optical sectioning by optical scanning holography and a Wiener filter," Appl. Opt. 45, 872-879 (2006).

6. G. Indebetouw, "A posteriori quasi-sectioning of the threedimensional reconstructions of scanning holographic microscopy," J. Opt. Soc. Am. A 23, 2657-2661 (2006).

7. X. Zhang, E. Y. Lam, T. Kim, Y. S. Kim, and T.-C. Poon, "Blind sectional image reconstruction for optical scanning holography," Opt. Lett. 34, 3098-3100 (2009).

8. X. Zhang, E. Y. Lam, and T.-C. Poon, "Reconstruction of sectional images in holography using inverse imaging," Opt. Express 16, 17215-17226 (2008).

9. H. Kim, S.-W. Min, B. Lee, and T.-C. Poon, "Optical sectioning for optical scanning holography using phase-space filtering with Wigner distribution functions," Appl. Opt. 47, D164-D175 (2008).

10. E. Y. Lam, X. Zhang, H. Vo, T.-C. Poon, and G. Indebetouw, "Three-dimensional microscopy and sectional image reconstruction using optical scanning holography," Appl. Opt. 48, H113-H119 (2009).

11. G. Indebetouw, "Scanning holographic microscopy with spatially incoherent sources: reconciling the holographic advantage with the sectioning advantage," J. Opt. Soc. Am. A 26 , 252-258 (2009).

12. T.-C. Poon, "Optical scanning holography-a review of recent progress," J. Opt. Soc. Korea 13, 406-415 (2009).

13. G. Pedrini and H. J. Tiziani, "Short-coherence digital microscopy by use of a lensless holographic imaging system," Appl. Opt. 41, 4489-4496 (2002).

14. P. Massatsch, F. Charrière, E. Cuche, P. Marquet, and C. D. Depeursinge, "Time-domain optical coherence tomography with digital holographic microscopy," Appl. Opt. 44, 1806-1812 (2005).
15. L. Martínez-León, G. Pedrini, and W. Osten, "Applications of short-coherence digital holography in microscopy," Appl. Opt. 44, 3977-3984 (2005).

16. C. Remmersmann, S. Stürwald, B. Kemper, P. Langehanenberg, and G. von Bally, "Phase noise optimization in temporal phase-shifting digital holography with partial coherence light sources and its application in quantitative cell imaging," Appl. Opt. 48, 1463-1472 (2009).

17. M. K. Kim, "Tomographic three-dimensional imaging of a biological specimen using wavelength-scanning digital interference holography," Opt. Express 7, 305-310 (2000).

18. L. Yu and M. K. Kim, "Wavelength-scanning digital interference holography for tomographic three-dimensional imaging by using the angular spectrum method," Opt. Lett. 30, 2092-2094 (2005).

19. L. F. Yu and Z. P. Chen, "Improved tomographic imaging of wavelength scanning digital holographic microscopy by use of digital spectral shaping," Opt. Express 15, 878-886 (2007).

20. S. Tamano, Y. Hayasaki, and N. Nishida, "Phase-shifting digital holography with a low-coherence light source for reconstruction of a digital relief object hidden behind a lightscattering medium," Appl. Opt. 45, 953-959 (2006).

21. M. K. Kim and M. Potcoava, "Fingerprint biometry applications of digital holography and low-coherence interference microscopy," in Digital Holography and Three-Dimensional Imaging, OSA Technical Digest (CD) (Optical Society of America, 2009), paper DTuB4.

22. R. Martínez-Cuenca, L. Martínez-León, J. Lancis, G. MínguezVega, O. Mendoza-Yero, E. Tajahuerce, P. Clemente, and P. Andrés, "Diffractive pulse-front tilt for low-coherence digital holography," in Digital Holography and Three-Dimensional Imaging, OSA Technical Digest (CD) (Optical Society of America, 2010), paper JMA33.

23. T. Zhang and I. Yamaguchi, "Three-dimensional microscopy with phase-shifting digital holography," Opt. Lett. 23, 1221-1223 (1998).

24. J. W. Goodman, Statistical Optics (Wiley, 1985).

25. L. F. Yu, B. Rao, J. Zhang, J. P. Su, Q. Wang, S. G. Guo, and Z. P. Chen, "Improved lateral resolution in optical coherence tomography by digital focusing using two-dimensional numerical diffraction method," Opt. Express 15, 7634-7641 (2007).

26. J. W. Goodman, Introduction to Fourier Optics, 3rd ed. (Roberts, 2005)

27. Y.-L. Ho, Y.-H. Lin, I.-J. Tsai, F.-J. Hsieh, and H.-J. Tsai, "In vivo assessment of cardiac morphology and function in heart-specific green fluorescent zebra fish," J. Formosan Med. Assoc. 106, 181-186 (2007). 